\title{
Efficacy and tolerability of topiramate-augmentation therapy for schizophrenia: a systematic review and meta-analysis of randomized controlled trials
}

This article was published in the following Dove Press journal:

Neuropsychiatric Disease and Treatment

15 December 2016

Number of times this article has been viewed

\section{Yuji Okuyama* \\ Kazuto Oya* \\ Shinji Matsunaga \\ Taro Kishi \\ Nakao Iwata}

Department of Psychiatry, Fujita

Health University School of Medicine,

Toyoake, Japan

*These authors contributed equally to this work
Correspondence: Taro Kishi

Department of Psychiatry, Fujita Health University School of Medicine,

I-98 Dengakugakubo, Kutsukake-cho,

Toyoake, Aichi 470-I 192, Japan

Tel +8I 562939250

Fax +8I 562 93। 83।

Email tarok@fujita-hu.ac.jp
Abstract: This study aimed to perform a comprehensive meta-analysis of topiramate-augmentation therapy in patients with schizophrenia receiving antipsychotic agents. Data published up to June 20, 2016 were obtained from the PubMed, PsycINFO, and Cochrane Library databases. Twelve randomized controlled trials comparing topiramate to placebo or antipsychotic only were included ( $\mathrm{n}=676$ patients). The primary outcome was change in overall symptoms. Relative risk (RR) and standardized mean difference (SMD), along with 95\% confidence intervals, were calculated using random effects model for each outcome. Topiramate-augmentation therapy was superior to the control for decreasing overall symptoms (SMD $-0.55,95 \%$ confidence interval -0.86 to $-0.24 ; P=0.001 ; P^{2}=55 \%$, eight comparisons, $\mathrm{n}=380$ ), positive symptoms (SMD -0.4), negative symptoms (SMD -0.47), and Positive and Negative Syndrome Scale general subscale scores (SMD -0.67). Furthermore, topiramate-augmentation therapy decreased weight (SMD - 0.69) and body mass index (SMD -0.95) compared with the control. Topiramate was similar to the control with respect to discontinuation due to all causes (RR 1.19), inefficacy (RR 1.71), and adverse events (RR 1.09). Topiramate was associated with higher incidence of paresthesia (RR 2.67) and attention difficulty (RR 8.97) compared with the control. Our results seemed to suggest that topiramate-augmentation therapy improves the psychopathology of schizophrenia with good tolerability and has the additional advantage of weight maintenance. However, because there were some limitations (numbers of studies and patients included in the meta-analysis were small, some studies used completer analysis, Chinese studies were included in the meta-analysis, and studies that had a risk of bias were included in the meta-analysis) in this study, we cannot apply the results of this study in daily clinical practice.

Keywords: schizophrenia, topiramate, efficacy, safety, systematic review, meta-analysis

\section{Introduction}

Schizophrenia is characterized by positive symptoms, such as hallucinations and delusions, negative symptoms, such as abulia and autism, and cognitive impairments. ${ }^{1}$ At its 15-year follow-up, a Dutch cohort study of psychotic disorder reported that two-thirds of patients suffered at least one relapse. Of those patients, one in six failed to recover fully and one in ten committed suicide. ${ }^{2}$ A previous meta-analysis that compared relapse rates between antipsychotic and placebo groups at 7-12 months found that antipsychotics significantly reduced relapse rates at 1 year over placebo, measured by the number needed to treat to benefit, which was three. ${ }^{3}$ Nonetheless, it appears difficult to maintain drug adherence by outpatients, which leads to relapse and readmission to hospital. ${ }^{4}$ In an anonymous online-survey study $(n=113)$, patients with schizophrenia reported discontinuing medication for the following 
reasons: side effects $(80 \%)$, stigma $(31 \%)$, mistrusting the physician/therapist (31\%), and rejection of medication in general $(28 \%) .{ }^{5}$ Therefore, side effects are considered the cardinal reason for poor compliance or noncompliance with antipsychotic medication. ${ }^{6}$

A recent network meta-analysis comparing 15 antipsychotics to placebo found that antipsychotics were superior for reducing overall symptoms of schizophrenia. ${ }^{7}$ However, all individual effect sizes were moderate, with the exception of clozapine, which had a large effect size compared with placebo. ${ }^{7}$ On the other hand, olanzapine and clozapine have been demonstrated to have a higher risk of metabolic abnormalities compared with other second-generation antipsychotics. ${ }^{8}$ For example, olanzapine revealed a higher risk of weight gain compared with amisulpride, aripiprazole, asenapine, lurasidone, paliperidone, and risperidone. ${ }^{8}$ Risperidone was also associated with the need for more use of antiparkinsonian medication compared with clozapine, olanzapine, quetiapine, and ziprasidone. ${ }^{9}$ When comparing antipsychotics (regarding overall symptoms as efficacy, and weight gain as safety), because the effect size of efficacy is smaller than that of safety, such as weight gain, several guidelines for the management of schizophrenia have recommended that the safer antipsychotic should be used for patients with schizophrenia. ${ }^{10}$ However, not all patients with schizophrenia respond to the safer antipsychotics.

Topiramate is approved for the treatment of epilepsy and prophylaxis of migraine by the US Food and Drug Administration. ${ }^{11}$ A previous review suggested that topiramate effects on psychopathology may be mediated through glutamatergic neurons, especially those expressing kainic acid and $\alpha$-amino-3-hydroxy-5-methylisoxazole-4-propionic acid (AMPA) receptors, and by inhibition of nitric oxide production. ${ }^{12}$ Although the mechanisms for improving metabolic disturbances are unclear, a previous report demonstrated that topiramate enhances insulin action and glucose transport in adipose cells from obese and insulin-resistant rodents. ${ }^{13}$ Further, appetite suppression through hypothalamic AMPA-receptor antagonism is thought to contribute to weight loss. ${ }^{14}$

To our knowledge, 12 randomized controlled trials (RCTs) of topiramate have been conducted for the treatment of schizophrenia and related psychoses. ${ }^{15-26}$ There were inconsistent results among these RCTs, however, regarding psychopathology and metabolic outcomes. Although four studies ${ }^{15,19,21,25}$ reported that topiramate was superior to control in improving psychopathological outcomes, six studies $^{16-18,20,23,26}$ reported that topiramate was similar to the control in this aspect, and the remaining studies ${ }^{22,24}$ did not report any psychopathological outcomes. To date, two meta-analyses have evaluated topiramate for the treatment of schizophrenia. ${ }^{27,28}$ Sommer et $\mathrm{al}^{27}$ reviewed three RCTs, ${ }^{15,20,23}$ and reported that topiramate augmentation for clozapine showed trend-level improvement regarding overall symptoms compared with placebo. Mizuno et $\mathrm{al}^{28}$ conducted a metaanalysis of multiple pharmacological strategies to combat weight gain in antipsychotic-treated schizophrenia patients that included two RCTs ${ }^{19,21}$ of topiramate augmentation. The pooled result showed that topiramate significantly lowered weight compared with the placebo (mean difference $-5.20 \mathrm{~kg}) .{ }^{28}$ However, these meta-analyses focused on topiramate augmentation as a strategy only for psychopathology or metabolic adverse events (including weight gain), and the number of topiramate trials included was small. In this updated systematic review and meta-analysis of topiramateaugmentation therapy for patients with schizophrenia and its related disorders, we have incorporated all relevant findings involving various antipsychotics from the 12 published RCTs. ${ }^{15-26}$ Because a meta-analysis can increase the statistical power for group comparisons and overcome the limitation of sample size when larger trials are lacking (http://www. cochrane.org), a systematic review and meta-analysis is considered to provide the "best evidence" for clinical practice. Moreover, safety outcomes are critical for adherence; therefore, we also evaluated topiramate-discontinuation rates and individual adverse events.

\section{Materials and methods Inclusion criteria, search strategy, data extraction, and outcomes}

This meta-analysis was performed according to the Preferred Reporting Items for Systematic Reviews and Meta-Analyses (PRISMA) guidelines. ${ }^{29} \mathrm{We}$ performed a systematic literature review according to the PICO (patients, intervention, comparison, outcome) strategy: patients, schizophrenia and schizophrenia-related disorders; intervention, topiramate in addition to an antipsychotic agent; comparison, versus placebo in addition to an antipsychotic agent or antipsychotic only; and outcome, primary outcome (improvement in overall symptoms) and secondary outcomes (improvement in positive and negative symptoms and Positive and Negative Syndrome Scale [PANSS] general subscale scores, ${ }^{30}$ decreasing weight and body mass index [BMI], improvement in Clinical Global Impression - Severity [CGI-S], ${ }^{31}$ and depressive symptoms, discontinuation rate, and individual adverse events). 
Only those RCTs involving the use of topiramate in patients with schizophrenia and its related disorders were included.

Relevant studies were identified through searches of PubMed, the Cochrane Library, and PsycINFO citations. There were no language restrictions, and we accepted studies retrieved using the keywords "topiramate" and "schizophrenia" published up to June 20, 2016. Additional eligible studies were sought by scrutiny of the reference lists from primary articles and relevant reviews. Two authors (YO and $\mathrm{KO}$ ) checked the inclusion and exclusion criteria for each of the identified studies, and resolved discrepancies in coding by discussion. The same authors independently extracted, checked, and entered data into RevMan version 5.3 for Windows (Cochrane Collaboration, London, UK). When data required for the meta-analysis were missing, the first/corresponding authors were contacted for additional information. We also assessed the risk of bias in the trials using the Cochrane risk-of-bias criteria (domains of randomsequence generation, blinding of participants and personnel, blinding of outcome assessment, incomplete outcome data, selective reporting, and other sources of bias) (http://www. cochrane.org).

\section{Data synthesis}

The primary efficacy measure was overall symptom reduction as measured by PANSS total scores and Brief Psychiatric Rating Scale (BPRS) ${ }^{32}$ total scores (Table 1). Secondary outcomes were as follows: 1) positive symptoms as measured by PANSS positive subscale scores and Scale for the Assessment of Positive Symptoms ${ }^{33}$ (Table 1), 2) negative symptoms as measured by PANSS negative subscale scores and Scale for the Assessment of Negative Symptoms ${ }^{34}$ (Table 1), 3) PANSS general subscale scores (Table 1), 4) CGI-S ${ }^{31}$ (Table 1), 5) depressive symptoms as measured by the Calgary Depression Scale for Schizophrenia ${ }^{35}$ or Montgomery-Åsberg Depression Rating Scale ${ }^{36}$ (Table 1), 6) weight (Table 1), 7) BMI (Table 1), and discontinuation rates due to 8) all causes, 9) inefficacy, and 10) adverse events.

In addition, we pooled the data for individual adverse events. With regard to tolerability, discontinuations due to worsening mental condition and unsatisfactory response ${ }^{17,23}$ were considered "discontinuation due to inefficacy", and discontinuation due to low leukocyte levels ${ }^{23}$ was considered "discontinuation due to adverse events". For estimation of missing data from individual studies, we imputed the standard deviation (SD) of BPRS-change scores from baseline to end point in Ko et al, ${ }^{19}$ from the data of Muscatello et al, ${ }^{20}$ and SD of CGI-S and BMI-change scores from baseline to

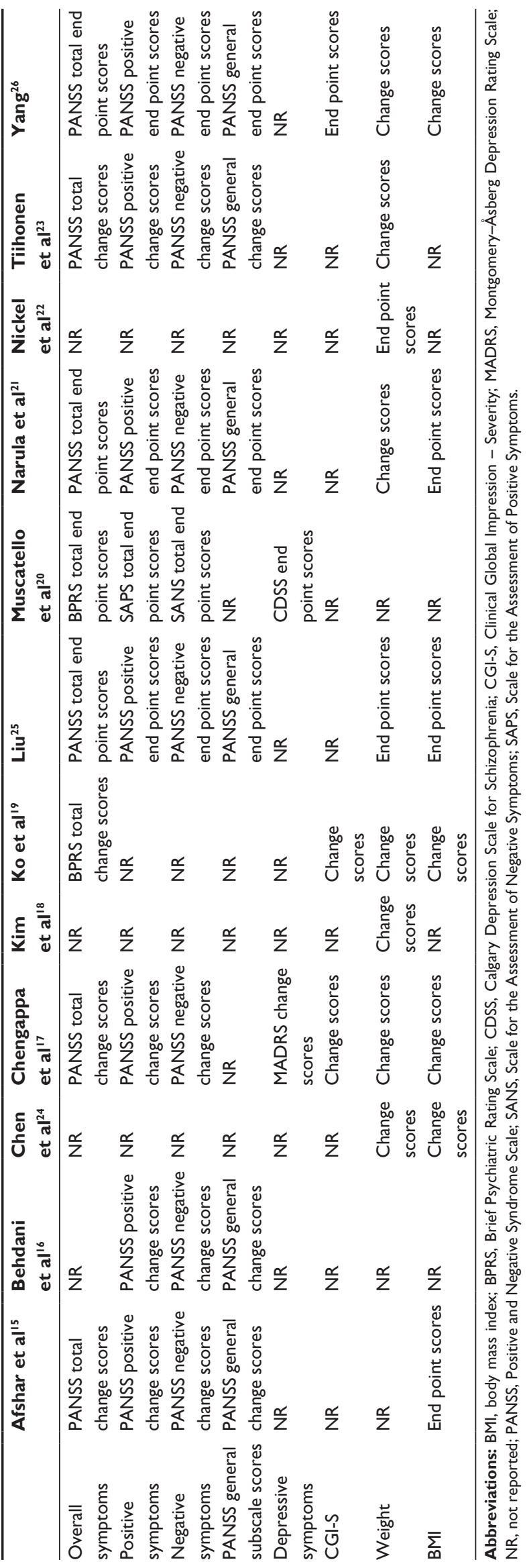


end point in the study of Ko et $\mathrm{al}^{19}$ from the SD of Chengappa et al. ${ }^{17}$ We converted weights from pounds to kilograms for Chengappa et al. ${ }^{17} \mathrm{We}$ assumed that the number of patients required for evaluating efficacy outcomes in Behdani et $\mathrm{al}^{16}$ was the same as the number of patients required for evaluating safety outcomes and the number of patients required for evaluating efficacy outcomes in Afshar et $\mathrm{al}^{15}$ was the same number of randomized patients for each treatment arm, because the trial used general linear model analysis. We used those estimations to increase sample size for analysis to gain as much statistical power as possible. The median highest topiramate dose for all studies except Ko et $\mathrm{al}^{19}$ was $238 \mathrm{mg} /$ day, so data from the $200 \mathrm{mg} /$ day topiramate group but not the $100 \mathrm{mg} /$ day topiramate group were used for meta-analysis. For individual adverse events, we considered memory disturbances ${ }^{23}$ and forgetfulness ${ }^{17}$ as "memory loss", and sedation ${ }^{17,23}$ and drowsiness ${ }^{16}$ as "somnolence".

\section{Statistical analysis}

We based our data analyses on intention to treat, observed cases, and crossover studies. This meta-analysis was performed using RevMan. To combine studies, we used the random-effect model described by DerSimonian and Laird. ${ }^{37}$ We used this conservative model to address the possibility that underlying effects differed across studies and populations were heterogeneous. For continuous data, we used standardized mean difference (SMD), combining effect size (Hedges' $g$ ) data and 95\% confidence interval (CI). For dichotomous data, relative risk (RR) was estimated along with the $95 \%$ CI. When the random-effect model revealed significant between-group differences in dichotomous outcomes, the number needed to harm $(\mathrm{NNH})$ was calculated. Study heterogeneity was measured using $\chi^{2}$ and $I^{2}$ statistics, with values of $P<0.05$ and $I^{2} \geq 50 \%$ indicating heterogeneity. ${ }^{38}$ In cases where $I^{2}$ values were $\geq 50 \%$ for primary outcome, sensitivity analyses were performed to determine the reasons for heterogeneity. Finally, funnel plots were visually inspected to explore the possibility of publication bias.

\section{Results}

\section{Study characteristics}

Searches of the PubMed, Cochrane Library, and PsycINFO databases yielded 116 hits. We excluded eight duplicate studies, 81 studies based on title or abstract review, and 20 studies after full-text reading. Five additional articles ${ }^{17,22,24-26}$ were identified by manually searching the review articles. ${ }^{17,39}$ Finally, 12 eligible studies ${ }^{15-26}$

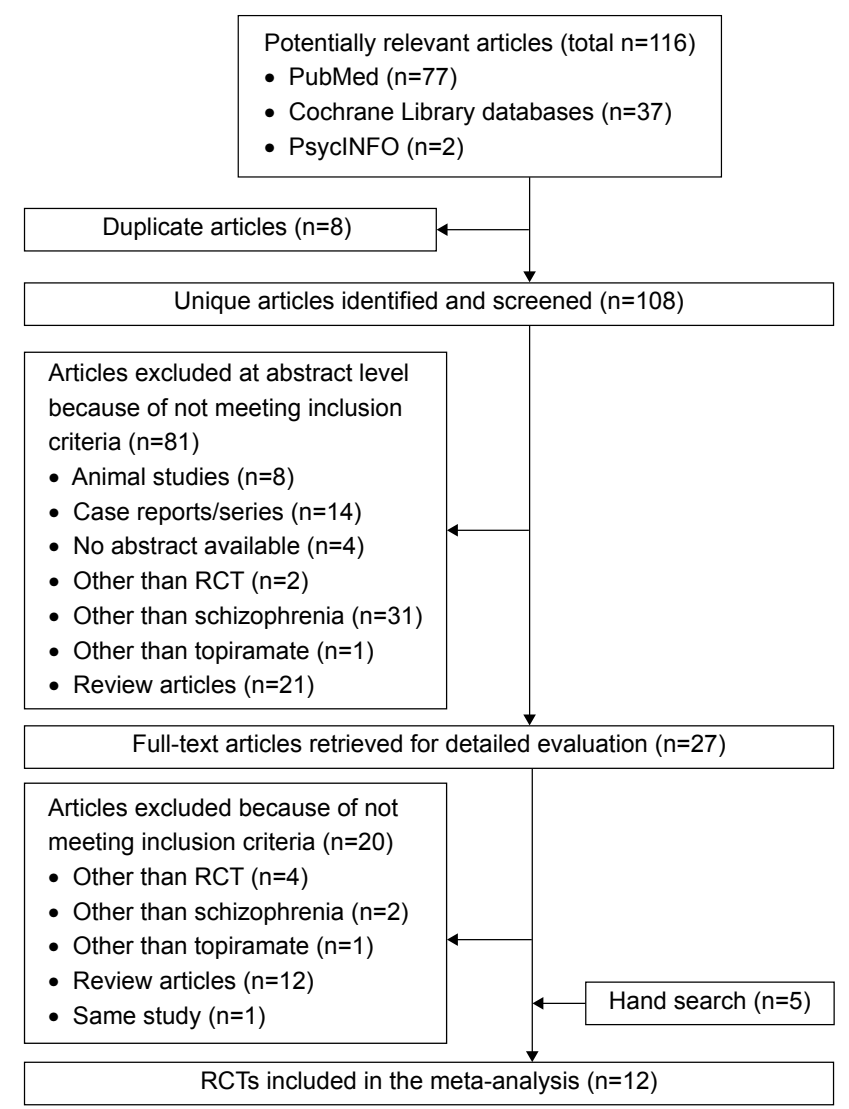

Figure I Literature search results.

Abbreviation: RCT, randomized controlled trial.

were accepted (Figure 1). Mean study duration was 13.4 (range 8-24) weeks, and 676 patients were included (schizophrenia 579, schizoaffective disorder 48, psychosis and unipolar or bipolar disorder 49). Patient numbers in each treatment arm were 358 for topiramate and 318 for control (consisting of 258 placebo-treated and 60 treatment as usual). The mean number of patients included in the individual studies was 56 . Three studies were published in Chinese. ${ }^{24-26}$ We evaluated the methodological quality of the individual studies based on Cochrane risk-of-bias criteria (Figures S1 and S2). Three studies did not employ a placebo control. ${ }^{18,24,25}$ One study was a crossover design. ${ }^{23}$ Seven studies used a completer analysis. ${ }^{19-22,24-26}$ Five studies were at high risk of selective reporting bias. ${ }^{15,18,20,21,24}$ One study was sponsored by the pharmaceutical industry. ${ }^{15}$ The characteristics of the studies are summarized in Table 2.

\section{Meta-analysis}

\section{Efficacy}

Topiramate as augmentation to antipsychotic therapy was superior to control (antipsychotic alone or placebo plus antipsychotic) for the reduction of overall symptoms (SMD -0.55 , 


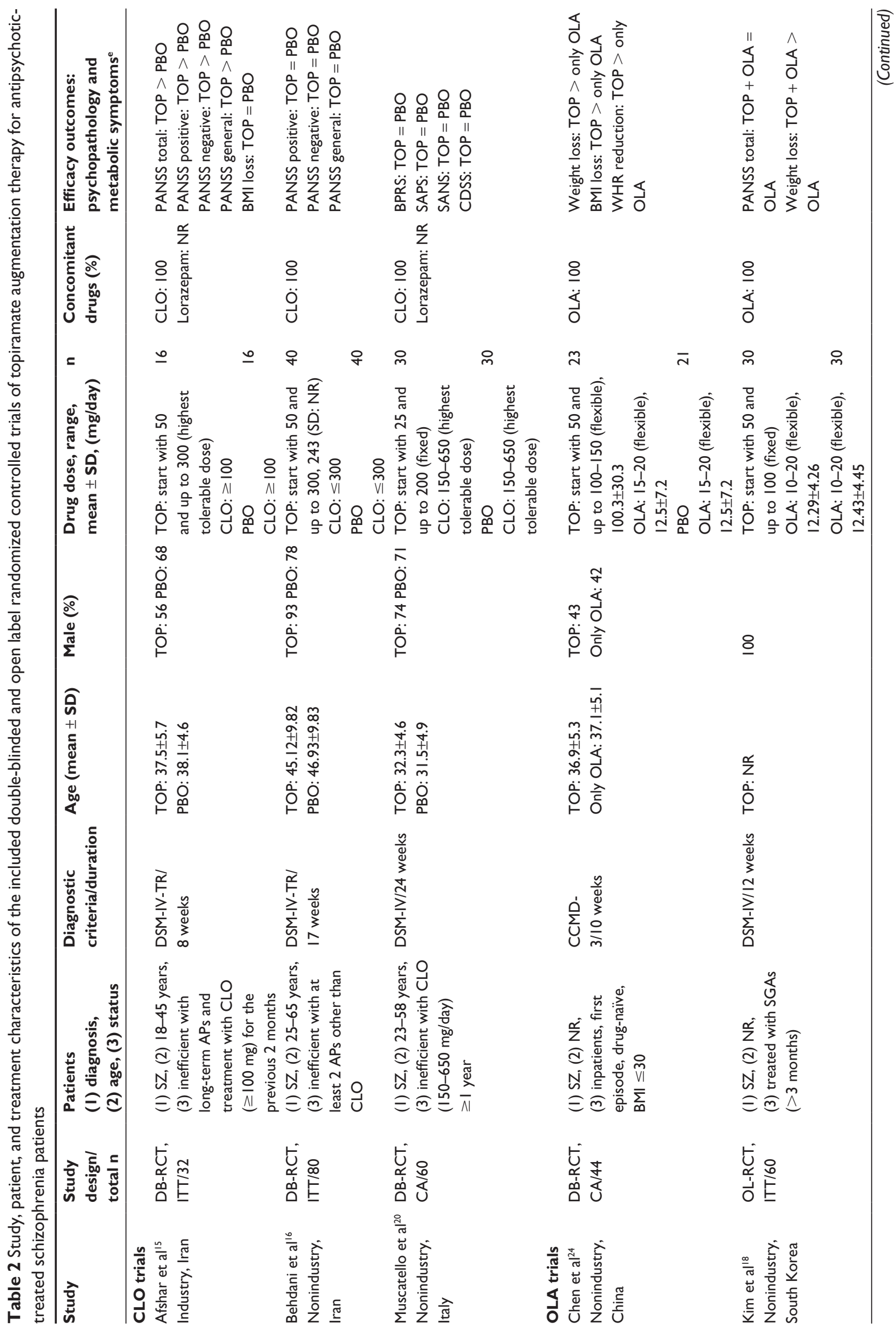




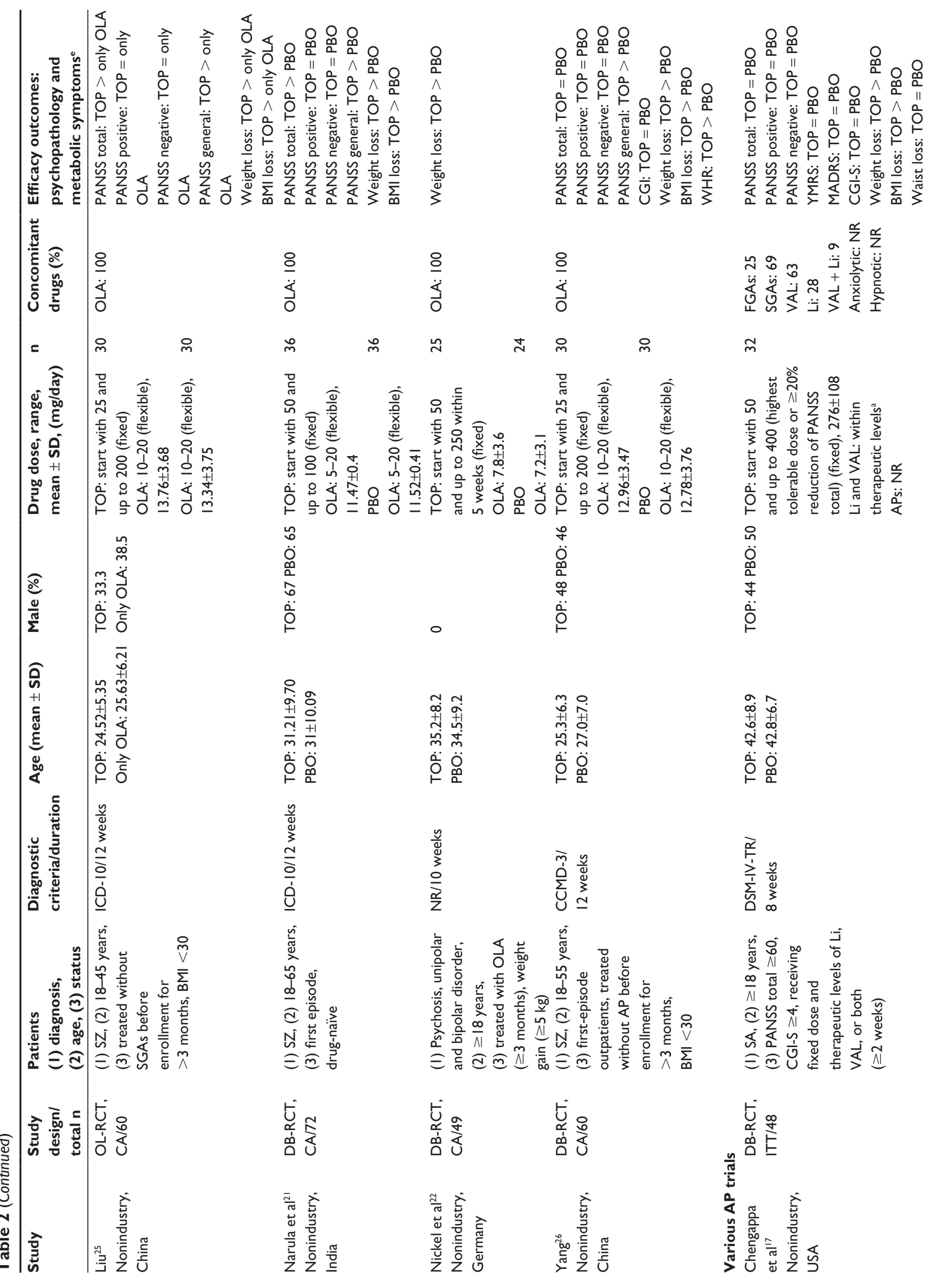




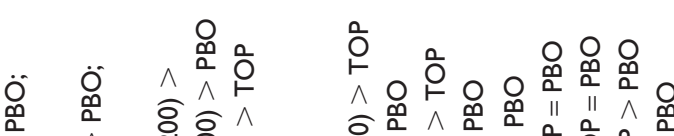
क्ष

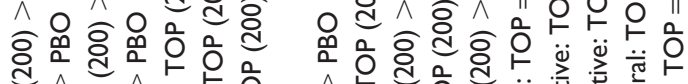

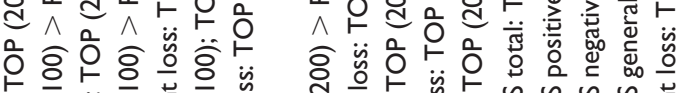

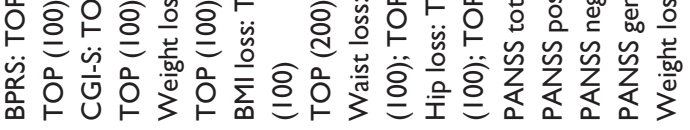

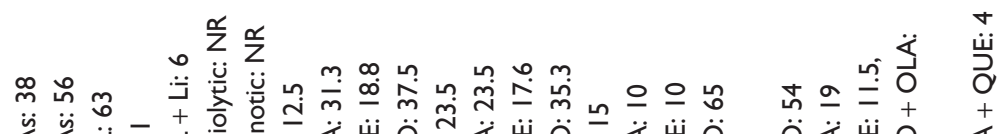

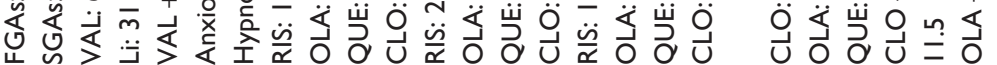

$\underline{-}$

i

ส

สิ

ส

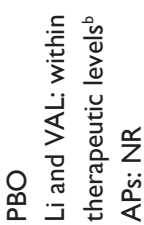

N

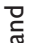

尊

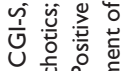

要

흐을

空紫言

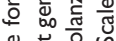

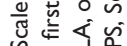

c

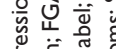

离.

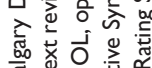

is.

岁产

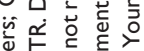

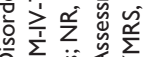

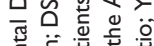

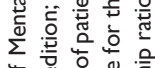

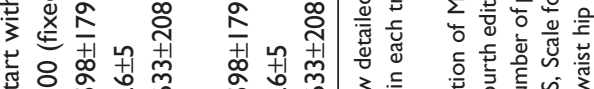

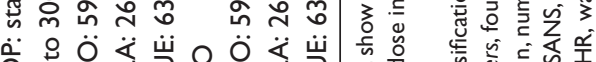

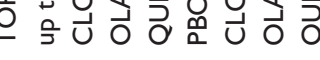

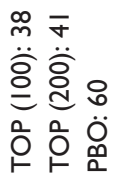

$\bar{\infty}$

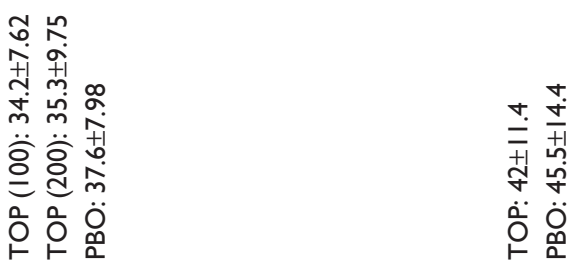

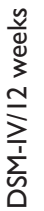

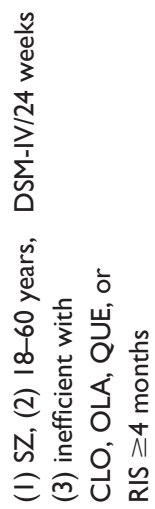

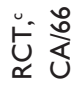

岗苍藏
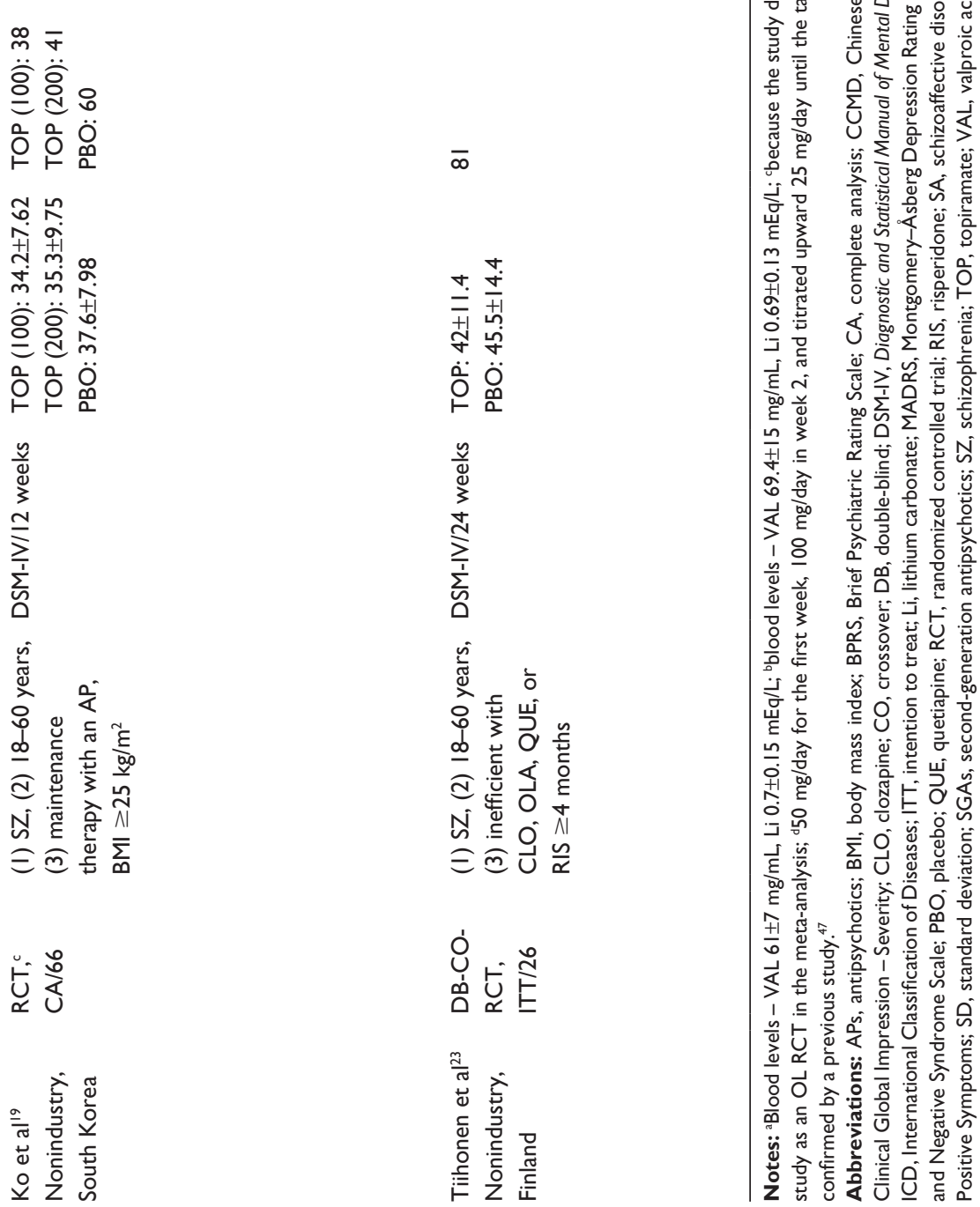
$95 \% \mathrm{CI}-0.86$ to $-0.24 ; P=0.001 ; I^{2}=55 \%$, eight comparisons, $\mathrm{n}=380$ ) (Figure 2A). Visual inspection of the funnel plot for primary outcome did not suggest publication bias (Figure S3). Topiramate was superior to control for the following secondary outcomes: positive symptoms (SMD $-0.4,95 \%$ CI -0.63 to $-0.17 ; P=0.001 ; I^{2}=29 \%$, eight comparisons, $\mathrm{n}=423$ ) (Figure $2 \mathrm{~B}$ ), negative symptoms (SMD $-0.47,95 \% \mathrm{CI}-0.82$ to $-0.12 ; P=0.01 ; I^{2}=67 \%$, eight comparisons, $\mathrm{n}=423$ ) (Figure $2 \mathrm{C}$ ), PANSS general subscale scores (SMD $-0.67,95 \% \mathrm{CI}-1.06$ to $-0.28 ; P=0.001$; $I^{2}=66 \%$, six comparisons, $\mathrm{n}=332$ ) (Figure 3A), weight loss (SMD $-0.69,95 \%$ CI -0.88 to $-0.49 ; P<0.0001 ; P^{2}=32 \%$, nine comparisons, $\mathrm{n}=447$ ) (Figure $3 \mathrm{~B}$ ), and $\mathrm{BMI}$ reduction (SMD $-0.95,95 \%$ CI -1.23 to $-0.66 ; P<0.0001 ; I^{2}=32 \%$, seven comparisons, $\mathrm{n}=331$ ) (Figure 3C). Topiramate was not different from control for depressive symptoms (SMD 0.15, $95 \% \mathrm{CI}-0.71$ to $1.01 ; P=0.73 ; I^{2}=75 \%$, two comparisons, $\mathrm{n}=91$ ) or CGI-S (SMD $-0.24,95 \% \mathrm{CI}-0.65$ to $0.18 ; P=0.26$; $r^{2}=31 \%$, three comparisons, $\mathrm{n}=140$ ).

\section{Sensitivity/subgroup analysis}

There was significant heterogeneity in overall symptoms among studies $\left(I^{2}=55 \%, P=0.03\right)$, so we performed 13 sensitivity analyses for primary outcome (antipsychotic class, blinding, analyzed population, region, control, study duration, topiramate dose, publication year, diagnosis, number of patients, patient status, language, and sponsorship) (Table 3). Significant heterogeneity in overall symptoms among the

A Study Statistics

Overall symptoms

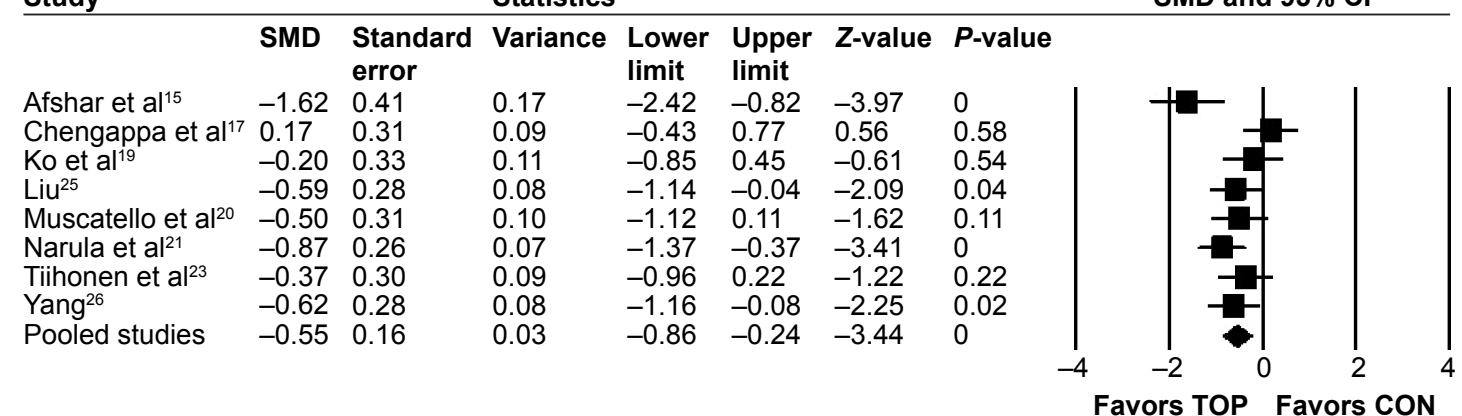

B

Study

Positive symptoms

Statistics

\begin{tabular}{|c|c|c|}
\hline Afshar et al ${ }^{15}$ & -1.17 & 0.38 \\
\hline Behdani et al $^{16}$ & -0.29 & 0.22 \\
\hline Chengappa et $\mathrm{al}^{17}$ & -0.02 & 0.31 \\
\hline $\operatorname{Liu}^{25}$ & -0.55 & 0.2 \\
\hline Muscatello et $\mathrm{al}^{20}$ & -0.84 & 0.32 \\
\hline Narula et $\mathrm{al}^{21}$ & -0.41 & 0.25 \\
\hline Tiihonen et al ${ }^{23}$ & -0.03 & 0.3 \\
\hline Yang $^{26}$ & & 0.2 \\
\hline Pooled studies & -0.40 & 0.1 \\
\hline
\end{tabular}

C

Study

Negative symptoms

Statistics

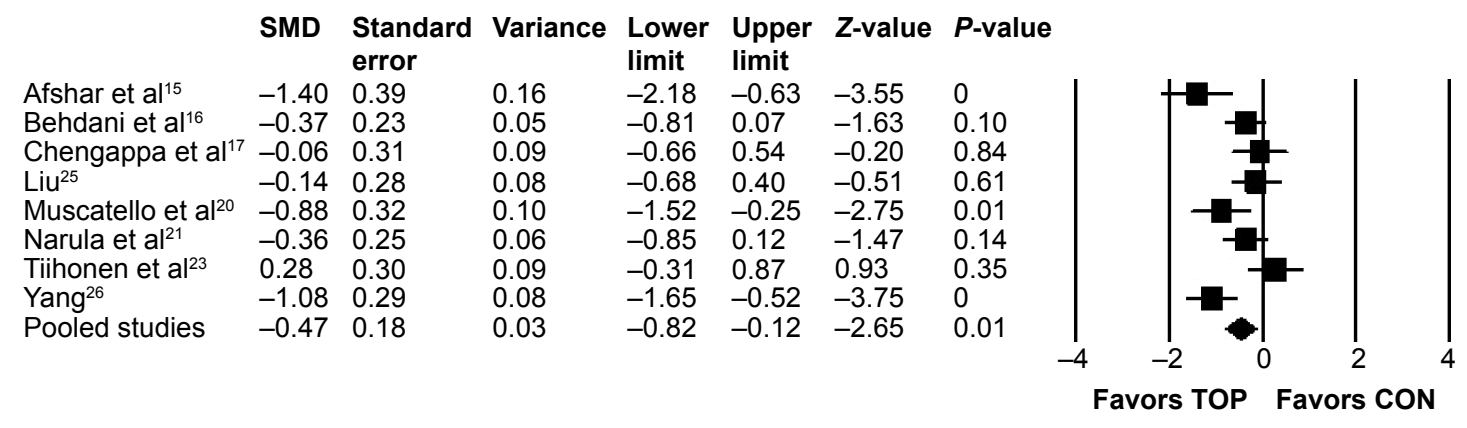

SMD and $95 \% \mathrm{Cl}$

$\begin{array}{lllll}\text { d Variance } & \begin{array}{l}\text { Lower } \\ \text { limit }\end{array} & \begin{array}{l}\text { Upper } \\ \text { limit }\end{array} & \text { Z-value } & \boldsymbol{P} \text {-value } \\ 0.15 & -1.92 & -1.42 & -3.05 & 0 \\ 0.05 & -0.73 & 0.15 & -1.28 & 0.20 \\ 0.09 & -0.62 & 0.58 & -0.08 & 0.94 \\ 0.08 & -1.10 & 0 & -1.96 & 0.05 \\ 0.10 & -1.47 & -0.22 & -2.63 & 0.01 \\ 0.06 & -0.89 & 0.07 & -1.66 & 0.10 \\ 0.09 & -0.62 & 0.55 & -0.12 & 0.91 \\ 0.07 & -0.76 & 0.30 & -0.85 & 0.40 \\ 0.01 & -0.63 & -0.17 & -3.35 & 0\end{array}$

Favors TOP Favors CON

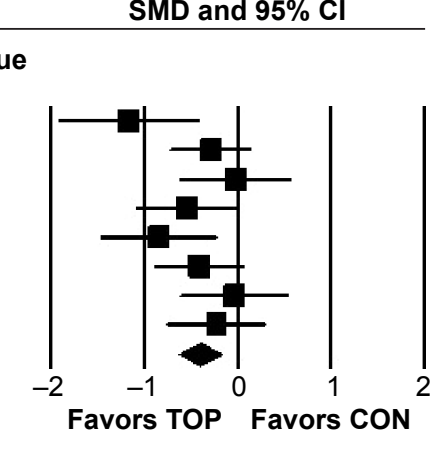

SMD and $95 \% \mathrm{Cl}$

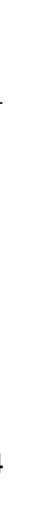

Figure 2 Forest plots of psychopathological outcomes.

Abbreviations: SMD, standardized mean difference; $\mathrm{Cl}$, confidence interval; TOP, topiramate; CON, control. 
various-antipsychotic subgroup, open-label subgroup, Europe-US subgroup, and older publication year (before 2008) subgroup disappeared without significant difference when comparing topiramate to control. On the other hand, significant heterogeneity in the olanzapine subgroup, non-intention-totreat population subgroup, Asia subgroup, the long-duration subgroup (13 weeks or more), low-dose topiramate subgroup (200 mg/day or less), newer publication year (after 2008) subgroup, diagnosis (schizophrenia) subgroup, not treatmentrefractory subgroup, and large number of patients (more than 55) subgroup disappeared when comparing topiramate to control. One study was unique, because it was the only study that was sponsored by the pharmaceutical industry (Table 3).

\section{Safety}

Topiramate and control did not differ regarding discontinuation rate due to all causes (RR 1.19, 95\% CI 0.79-1.79; $P=0.4 ; I^{2}=1 \%$, ten comparisons, $\left.\mathrm{n}=561\right)$, discontinuation rate due to inefficacy (RR 1.71, 95\% CI 0.52-5.66; $P=0.38$; $I^{2}=0$, seven comparisons, $\mathrm{n}=377$ ), or discontinuation rate due to adverse events (RR 1.09, 95\% CI 0.14-8.49; $P=0.93$; $I^{2}=0$, seven comparisons, $\mathrm{n}=377$ ). Although topiramate was associated with a lower incidence of weight gain (RR 0.31 , $95 \%$ CI $0.15-0.64 ; P=0.002 ; I^{2}=62 \%, \mathrm{NNH}-2$, three comparisons, $n=165$ ) (Figure $4 A$ ), $\geq 7 \%$ weight gain (RR 0.25 , $95 \%$ CI $0.12-0.51 ; P=0.0002 ; I^{2}=0, \mathrm{NNH}-2$, two comparisons, $n=97$ ) (Figure 4B), and increased appetite (RR 0.26,
A Study

\begin{tabular}{llll}
\hline & SMD & $\begin{array}{l}\text { Standard } \\
\text { error }\end{array}$ & Variance \\
Afshar et $\mathrm{al}^{15}$ & -0.99 & 0.37 & 0.14 \\
${\text { Behdani et } \mathrm{al}^{16}}^{16}$ & 0.08 & 0.22 & 0.05 \\
Liu $^{25}$ & -0.61 & 0.28 & 0.08 \\
Narula et al $^{21}$ & -1.03 & 0.26 & 0.07 \\
${\text { Tiihonen et } \mathrm{al}^{23}}^{23}$ & -0.71 & 0.31 & 0.09 \\
Yang $^{26}$ & -0.92 & 0.28 & 0.08 \\
Pooled studies & -0.67 & 0.20 & 0.04
\end{tabular}

B

Study Statistics

Statistics
PANSS general subscale scores SMD and $95 \% \mathrm{CI}$

$\begin{array}{llll}\begin{array}{l}\text { Lower } \\ \text { limit }\end{array} & \begin{array}{l}\text { Upper } \\ \text { limit }\end{array} & \text { Z-value } & P \text {-value } \\ -1.73 & -0.26 & -2.65 & 0.01 \\ -0.36 & 0.52 & 0.37 & 0.71 \\ -1.16 & -0.06 & -2.16 & 0.03 \\ -1.54 & -0.52 & -3.96 & 0 \\ -1.32 & -0.11 & -2.32 & 0.02 \\ -1.48 & -0.36 & -3.24 & 0 \\ -1.06 & -0.28 & -3.40 & 0\end{array}$

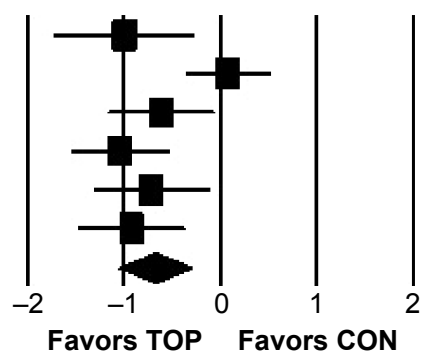

SMD and $95 \% \mathrm{CI}$

Chen et al
Chengappa et $\mathrm{al}^{17}$
Kim et al ${ }^{18}$
Ko et al
Liu
Narula et $\mathrm{al}^{21}$
Nickel et $\mathrm{al}^{22}$
Tiihonen et al
Yang
Pooled studies

$\begin{array}{lll}\text { SMD } & \begin{array}{l}\text { Standard } \\ \text { error }\end{array} & \text { Variance } \\ -0.86 & 0.33 & 0.11 \\ -0.89 & 0.33 & 0.11 \\ -0.62 & 0.26 & 0.07 \\ -0.39 & 0.33 & 0.11 \\ -0.80 & 0.29 & 0.08 \\ -0.56 & 0.25 & 0.06 \\ -0.38 & 0.31 & 0.10 \\ -0.28 & 0.30 & 0.09 \\ -1.50 & 0.31 & 0.09 \\ -0.69 & 0.10 & 0.01\end{array}$

C

Study

Statistics

\section{$B M I$ reduction}

$\begin{array}{llll}\begin{array}{l}\text { Lower } \\ \text { limit }\end{array} & \begin{array}{l}\text { Upper } \\ \text { limit }\end{array} & \text { Z-value } & \boldsymbol{P} \text {-valu } \\ -1.50 & -0.22 & -2.64 & 0.01 \\ -1.54 & -0.23 & -2.65 & 0.01 \\ -1.14 & -0.10 & -2.35 & 0.02 \\ -1.04 & 0.27 & -1.16 & 0.25 \\ -1.36 & -0.24 & -2.80 & 0.01 \\ -1.05 & -0.07 & -2.24 & 0.03 \\ -0.99 & 0.23 & -1.21 & 0.23 \\ -0.86 & 0.31 & -0.92 & 0.36 \\ -2.09 & -0.90 & -4.90 & 0 \\ -0.88 & -0.49 & -6.95 & 0\end{array}$

$\begin{array}{llllllll} & \text { SMD } & \begin{array}{l}\text { Standard } \\ \text { error }\end{array} & \text { Variance } & \begin{array}{l}\text { Lower } \\ \text { limit }\end{array} & \begin{array}{l}\text { Upper } \\ \text { limit }\end{array} & \text { Z-value } & \begin{array}{l}\boldsymbol{P} \text {-value } \\ \text { Afshar et al }\end{array} \\ {\text { Chen et } \mathrm{al}^{24}}^{15} & -0.53 & 0.36 & 0.13 & -1.23 & 0.18 & -1.47 & 0.14 \\ \text { Chengappa et al }^{17} & -1.28 & 0.34 & 0.12 & -1.96 & -0.61 & -3.74 & 0 \\ \text { Ko et al }^{19} & -0.93 & 0.33 & 0.11 & -1.58 & -0.27 & -2.77 & 0.01 \\ \text { Liu }^{25} & -1.04 & 0.35 & 0.12 & -1.72 & -0.35 & -2.95 & 0 \\ \text { Narula et al }^{21} & -0.76 & 0.28 & 0.08 & -1.32 & -0.21 & -2.69 & 0.01 \\ \text { Yang }^{26} & -0.60 & 0.25 & 0.06 & -1.09 & -0.12 & -2.42 & 0.02 \\ \text { Pooled studies } & -1.58 & 0.31 & 0.10 & -2.18 & -0.97 & -5.11 & 0 \\ & -0.95 & 0.14 & 0.02 & -1.23 & -0.66 & -6.55 & 0\end{array}$

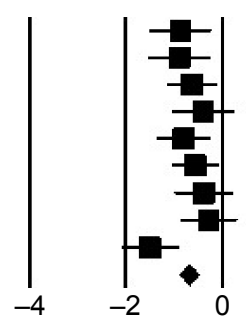

Favors TOP Favors CON

SMD and $95 \% \mathrm{Cl}$

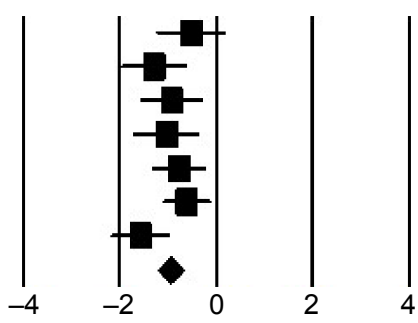

Favors TOP Favors CON

Figure 3 Forest plots of psychopathological and anthropometric outcomes.

Abbreviations: PANSS, Positive and Negative Syndrome Scale; SMD, standardized mean difference; Cl, confidence interval; TOP, topiramate; CON, control; BMI, body mass index. 
Table 3 Sensitivity analysis for overall symptoms of topiramate-augmentation therapy

\begin{tabular}{|c|c|c|c|c|c|c|c|c|c|}
\hline \multirow[t]{2}{*}{ Variable } & \multirow[t]{2}{*}{ Subgroup } & \multirow[t]{2}{*}{ Studies (n) } & \multirow[t]{2}{*}{ Patients (n) } & \multirow[t]{2}{*}{$I^{2}(\%)$} & \multirow[t]{2}{*}{ SMD } & \multirow[t]{2}{*}{$95 \% \mathrm{Cl}$} & \multirow[t]{2}{*}{$P$-value } & \multicolumn{2}{|c|}{$\begin{array}{l}\text { Test for } \\
\text { subgroup } \\
\text { differences }\end{array}$} \\
\hline & & & & & & & & $P$-value & $I^{2}(\%)$ \\
\hline Antipsychotic & Clozapine & 2 & 75 & 77 & -1 & -2.06 to 0.06 & 0.06 & 0.04 & 69.7 \\
\hline \multirow[t]{2}{*}{ class } & Olanzapine & 3 & 175 & 0 & -0.69 & -1 to -0.39 & $<0.00001$ & & \\
\hline & Various APs & 3 & 130 & 0 & -0.13 & -0.48 to 0.22 & 0.47 & & \\
\hline \multirow[t]{2}{*}{ Blinding } & Double-blind & 6 & 367 & 69 & -0.64 & -1.13 to -0.16 & 0.009 & 0.49 & 0 \\
\hline & Open & 2 & 90 & 0 & -0.42 & -0.84 to 0 & 0.05 & & \\
\hline Analyzed & ITT & 2 & 80 & 91 & -0.68 & -2.39 to 1.03 & 0.43 & 0.78 & 0 \\
\hline \multirow[t]{2}{*}{ population } & Non-ITT & 5 & 255 & 0 & -0.59 & -0.84 to -0.33 & $<0.00001$ & & \\
\hline & Crossover & 1 & 45 & NA & -0.36 & -0.95 to 0.23 & 0.23 & & \\
\hline \multirow[t]{2}{*}{ Region } & Asia & 5 & 244 & 47 & -0.72 & -1.09 to -0.36 & 0.0001 & 0.07 & 69.2 \\
\hline & Europe and USA & 3 & 136 & 23 & -0.23 & -0.62 to 0.17 & 0.26 & & \\
\hline \multirow[t]{2}{*}{ Control } & PBO & 7 & 327 & 59 & -0.54 & -0.89 to -0.18 & 0.003 & 0.9 & 0 \\
\hline & Non-PBO & I & 53 & NA & -0.58 & -1.13 to -0.03 & 0.04 & & \\
\hline Study & $<13$ weeks & 6 & 292 & 65 & -0.58 & -1 to -0.17 & 0.006 & 0.6 & 0 \\
\hline duration & $\geq 13$ weeks & 2 & 88 & 0 & -0.43 & -0.85 to 0 & 0.05 & & \\
\hline Topiramate & $\leq 200 \mathrm{mg}$ & 5 & 255 & 0 & -0.59 & -0.84 to -0.33 & $<0.00001$ & 0.95 & 0 \\
\hline dose & $>200 \mathrm{mg}$ & 3 & 125 & 83 & -0.55 & -1.47 to 0.36 & 0.24 & & \\
\hline Publication & $<2008$ & 3 & 130 & 0 & -0.13 & -0.48 to 0.22 & 0.47 & 0.008 & 85.7 \\
\hline year & $\geq 2008$ & 5 & 250 & 28 & -0.76 & -1.07 to -0.45 & $<0.00001$ & & \\
\hline \multirow[t]{2}{*}{ Diagnosis } & Schizophrenia & 7 & 332 & 32 & -0.63 & -0.91 to -0.36 & $<0.00001$ & 0.02 & 82.4 \\
\hline & Schizoaffective disorder & I & 48 & NA & 0.17 & -0.43 to 0.77 & 0.58 & & \\
\hline \multirow{2}{*}{$\begin{array}{l}\text { Number of } \\
\text { patients }\end{array}$} & $\mathrm{n} \leq 55$ & 3 & 125 & 83 & -0.55 & -1.47 to 0.36 & 0.24 & 0.95 & 0 \\
\hline & $\mathrm{n}>55$ & 5 & 255 & 0 & -0.59 & -0.84 to -0.33 & $<0.00001$ & & \\
\hline \multirow[t]{2}{*}{ Patient status } & Treatment-refractory & 3 & 120 & 68 & -0.76 & -1.44 to -0.08 & 0.03 & 0.41 & 0 \\
\hline & Not treatment-refractory & 5 & 260 & 48 & -0.44 & -0.8 to -0.09 & 0.01 & & \\
\hline \multirow[t]{2}{*}{ Language } & English & 6 & 272 & 66 & -0.53 & -0.96 to -0.09 & 0.02 & 0.81 & 0 \\
\hline & Chinese & 2 & 108 & 0 & -0.6 & -0.98 to -0.21 & 0.003 & & \\
\hline \multirow[t]{2}{*}{ Sponsorship } & Industry & I & 32 & NA & -1.58 & -2.38 to -0.77 & 0.0001 & 0.009 & 85.5 \\
\hline & Nonindustry & 7 & 348 & 24 & -0.45 & -0.7 to -0.2 & 0.0004 & & \\
\hline
\end{tabular}

Abbreviations: APs, antipsychotics; $\mathrm{Cl}$, confidence interval; ITT, intention to treat; NA, not applicable; PBO, placebo; SMD, standardized mean difference.

$95 \%$ CI $0.1-0.66 ; P=0.005 ; P=0, \mathrm{NNH}-4$, two comparisons, $n=120$ ) (Figure 4C) than the controls, it was associated with a higher incidence of paresthesia (RR 2.67, 95\% CI 1.15-6.19; $P=0.02 ; I^{2}=0$, NNH not significant, four comparisons, $\mathrm{n}=248$ ) (Figure 5A) and attention difficulty (RR 8.97, 95\% CI $1.17-68.63 ; P=0.03 ; I^{2}=0$, NNH 8 , two comparisons, $\mathrm{n}=120$ ) (Figure 5B) than the controls. There were no significant differences in individual adverse events reported (asthenia, constipation, diarrhea, dizziness, dry mouth, fatigue, headache, insomnia, memory loss, muscle weakness, nausea, psychomotor slowing, psychosis exacerbation, or somnolence) between topiramate and control (Table 4).

\section{Discussion}

To the best of our knowledge, the current study is the largest comprehensive systematic review and meta-analysis of topiramate-augmentation therapy for schizophrenia and related diseases. Topiramate augmentation was more efficacious than control (antipsychotic alone or antipsychotic plus placebo) for reducing overall, positive, and negative symptoms, PANSS general subscale scores, weight, and BMI. We did not find any cause for the heterogeneity among individual studies, despite performing 13 sensitivity analyses of overall symptoms. Based on the results of the sensitivity analyses, low-dose topiramate ( $\leq 200 \mathrm{mg} /$ day)-augmentation therapy for olanzapine may be useful. The prevalence of metabolic syndrome is at least twice as high in schizophrenia patients compared with the age-adjusted population. ${ }^{40}$ Given the importance of maintaining medication adherence for schizophrenia, it is often necessary to use a safer but less efficacious antipsychotic. Augmentation therapy should be considered before changing from the safer antipsychotic to one with greater potential efficacy but also higher metabolic risk. ${ }^{41,42}$ In a prospective study, $27 \%$ of patients with schizophrenia 
A

Weight gain

\begin{tabular}{|c|c|c|c|c|c|c|}
\hline \multirow[t]{2}{*}{ Study } & \multicolumn{5}{|c|}{ Statistics } & \multirow[t]{2}{*}{ Risk ratio and $95 \% \mathrm{Cl}$} \\
\hline & $\begin{array}{l}\text { Risk } \\
\text { ratio }\end{array}$ & $\begin{array}{l}\text { Lower } \\
\text { limit }\end{array}$ & $\begin{array}{l}\text { Upper } \\
\text { limit }\end{array}$ & Z-value & $P$-value & \\
\hline Liu $^{25}$ & 0.16 & 0.07 & 0.38 & -4.18 & 0 & \\
\hline Narula et $\mathrm{al}^{21}$ & 0.28 & 0.16 & 0.49 & -4.57 & 0 & \\
\hline Tiihonen et $\mathrm{al}^{23}$ & 0.75 & 0.28 & 2 & -0.58 & 0.56 & \\
\hline Pooled studies & 0.31 & 0.15 & 0.64 & -3.14 & 0 & \\
\hline
\end{tabular}

Favors TOP Favors CON

B

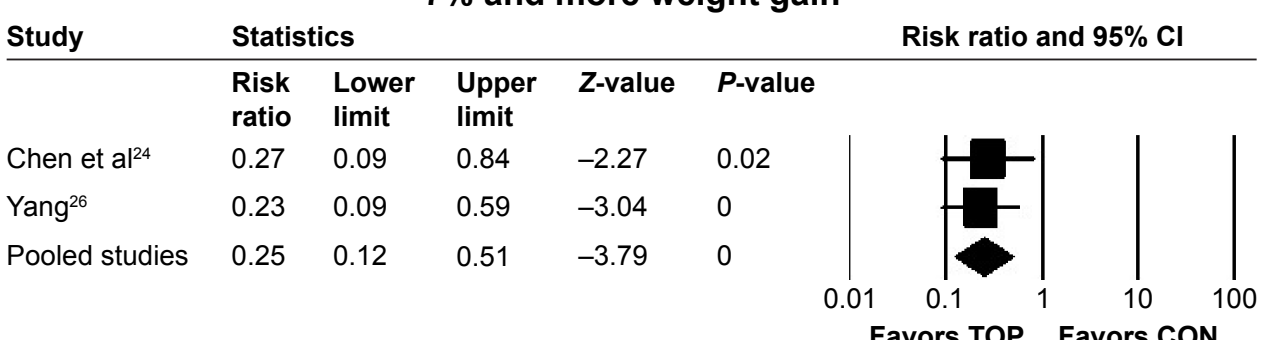

C

Increased appetite

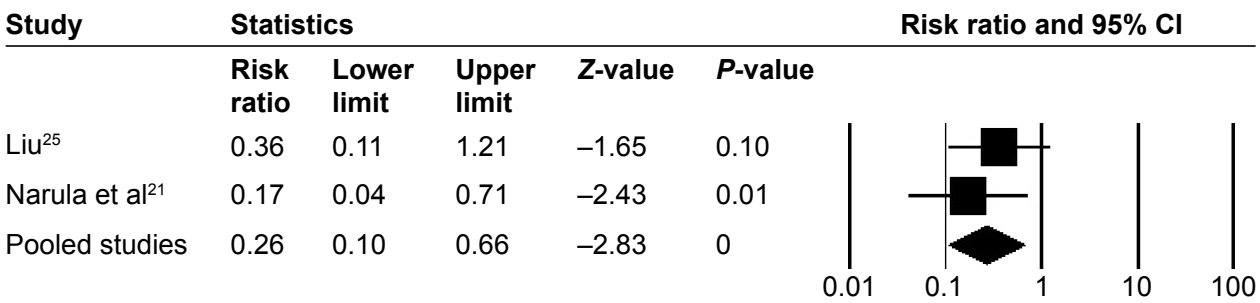

Favors TOP Favors CON

Figure 4 Forest plots of individual adverse events.

Abbreviations: $\mathrm{Cl}$, confidence interval; TOP, topiramate; $\mathrm{CON}$, control.

A

Paresthesia

Study

Statistics

Risk ratio and $95 \% \mathrm{Cl}$

$\begin{array}{lllll}\begin{array}{l}\text { Risk } \\ \text { ratio }\end{array} & \begin{array}{l}\text { Lower } \\ \text { limit }\end{array} & \begin{array}{l}\text { Upper } \\ \text { limit }\end{array} & \text { Z-value } & \boldsymbol{P} \text {-value } \\ 1.67 & 0.53 & 5.22 & 0.88 & 0.38 \\ 4.24 & 1.07 & 16.75 & 2.06 & 0.04 \\ 7.21 & 0.39 & 134.32 & 1.32 & 0.19 \\ 2.67 & 1.15 & 6.19 & 2.29 & 0.02\end{array}$

B

Attention difficulty

Study

Statistics

Risk ratio and $95 \% \mathrm{Cl}$

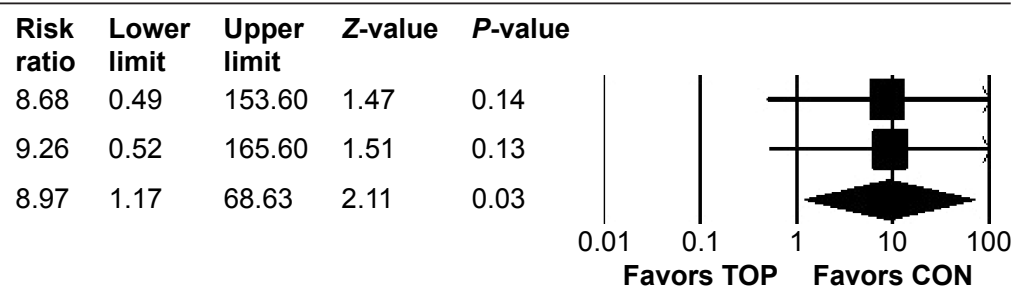

Figure 5 Forest plots of individual adverse events.

Abbreviations: $\mathrm{Cl}$, confidence interval; TOP, topiramate; $\mathrm{CON}$, control. 
Table 4 Discontinuation rates and individual adverse events

\begin{tabular}{|c|c|c|c|c|c|c|}
\hline & Studies (n) & Patients (n) & $I^{2}(\%)$ & Risk ratio & $95 \% \mathrm{Cl}$ & P-value \\
\hline Discontinuation due to inefficacy & 7 & 377 & 0 & I.7| & $0.52-5.66$ & 0.38 \\
\hline Discontinuation due to adverse events & 7 & 377 & 0 & 1.09 & $0.14-8.49$ & 0.93 \\
\hline Asthenia & 2 & 112 & 0 & 0.98 & $0.54-1.77$ & 0.95 \\
\hline Constipation & 3 & 195 & 49 & 0.43 & $0.05-3.68$ & 0.44 \\
\hline Diarrhea & 2 & 101 & 0 & I. 10 & $0.36-3.4$ & 0.87 \\
\hline Dizziness & 5 & 308 & 0 & 0.56 & $0.29-1.09$ & 0.09 \\
\hline Fatigue & 2 & 106 & 0 & 0.87 & $0.5 \mathrm{I}-\mathrm{I} .47$ & 0.59 \\
\hline Headache & 4 & 236 & 6 & 0.89 & $0.3-2.64$ & 0.83 \\
\hline Insomnia & 2 & 127 & 0 & 0.68 & $0.2-2.27$ & 0.53 \\
\hline Memory loss & 3 & 148 & 0 & 0.99 & $0.46-2.13$ & 0.97 \\
\hline Nausea & 4 & 241 & 0 & 0.55 & $0.2-1.5$ & 0.24 \\
\hline Psychomotor slowing & 2 & 120 & 0 & 3.30 & $0.58-18.93$ & 0.18 \\
\hline Psychosis exacerbation & 2 & 123 & NA & 5.00 & $0.25-100.97$ & 0.29 \\
\hline Somnolence & 6 & 348 & 4 & 1.09 & $0.81-1.45$ & 0.57 \\
\hline
\end{tabular}

Abbreviations: $\mathrm{Cl}$, confidence interval; NA, not applicable.

eventually developed poor adherence. ${ }^{43}$ Moritz et al ${ }^{44}$ studied the reasons for antipsychotic nonadherence, and concluded that side effects were the most common reason. Weight gain is also a well-known side effect, especially with secondgeneration antipsychotics, which are more widely used throughout the world than first-generation antipsychotics for treating schizophrenia. ${ }^{45}$ Furthermore, augmentation therapy for clozapine may be particularly important, since clozapine is thought to be the final option for treatment-resistant schizophrenia (alongside modified electroconvulsive therapy). If clozapine efficacy is still insufficient, effective augmentation drugs should be considered. ${ }^{27}$

However, we cannot apply the results of this study in daily clinical practice because of the following. There are some limitations to this meta-analysis. The main limitation is the paucity of RCTs, despite updating from previous metaanalyses. ${ }^{27,28}$ All the trials included were small, and it is well documented that small trials exaggerate effects. Second, although we utilized a funnel plot to assess potential publication bias, these are generally used only if ten or more studies are included. The third limitation is the short follow-up period (8-24 weeks). Fourth, we did not investigate the optimal topiramate dose for augmentation therapy. Future research should investigate the long-term efficacy of multiple doses and generate more safety data using larger samples. Fifth, although several of our included studies were from China, it has been reported that many Chinese studies are problematic. ${ }^{46}$ Some studies included in the meta-analysis did not report detailed information about sequence generation or allocation concealment. Moreover, seven studies used a completer analysis.

\section{Conclusion}

Our results indicate that topiramate-augmentation therapy is well tolerated, can improve the psychopathology of schizophrenia, and reduces weight gain associated with antipsychotics. Since there were some limitations in this study, we could not apply the results of this study in daily clinical practice. Future research should investigate long-term efficacy and generate more safety data for schizophrenia patients receiving topiramate augmentation of antipsychotics.

\section{Disclosure}

The authors report no conflicts of interest in this work, but report the following interests: Dr Okuyama has received speaker's honoraria from Janssen; Dr Oya has received honoraria from Eisai, Eli Lilly, Janssen, Meiji, Otsuka, and Tanabe-Mitsubishi; Dr Matsunaga has received honoraria from Eisai, Janssen, Novartis, Daiichi Sankyo, Ono, Eli Lilly, Takeda, and Otsuka and has a Fujita Health University School of Medicine research grant and Grant-in-Aid for Young Scientists (B); Dr Kishi has received speaker's honoraria from Abbvie, Astellas, Daiichi Sankyo, Dainippon Sumitomo, Eisai, Eli Lilly, GlaxoSmithKline, Janssen, Yoshitomi, Otsuka, Meiji, Mochida, Shionogi, TanabeMitsubishi, Tsumura, Novartis, and Pfizer and has a Fujita Health University School of Medicine research grant and Grant-in-Aid for Young Scientists (B); and Dr Iwata has received honoraria from Astellas, Dainippon Sumitomo, Eli Lilly, GlaxoSmithKline, Janssen, Yoshitomi, Otsuka, Meiji, Shionogi, Novartis, and Pfizer.

\section{References}

1. MacDonald AW, Schulz SC. What we know: findings that every theory of schizophrenia should explain. Schizophr Bull. 2009;35(3): 493-508.

2. Wiersma D, Nienhuis FJ, Slooff CJ, Giel R. Natural course of schizophrenic disorders: a 15-year followup of a Dutch incidence cohort. Schizophr Bull. 1998;24(1):75-85.

3. Leucht S, Tardy M, Komossa K, et al. Antipsychotic drugs versus placebo for relapse prevention in schizophrenia: a systematic review and meta-analysis. Lancet. 2012;379(9831):2063-2071. 
4. Valenstein M, Copeland LA, Blow FC, et al. Pharmacy data identify poorly adherent patients with schizophrenia at increased risk for admission. Med Care. 2002;40(8):630-639.

5. Moritz S, Favrod J, Andreou C, et al. Beyond the usual suspects: positive attitudes towards positive symptoms is associated with medication noncompliance in psychosis. Schizophr Bull. 2013;39(4): 917-922.

6. Haddad PM, Brain C, Scott J. Nonadherence with antipsychotic medication in schizophrenia: challenges and management strategies. Patient Relat Outcome Meas. 2014;5:43-62.

7. Leucht S, Cipriani A, Spineli L, et al. Comparative efficacy and tolerability of 15 antipsychotic drugs in schizophrenia: a multiple-treatments meta-analysis. Lancet. 2013;382(9896):951-962.

8. Rummel-Kluge C, Komossa K, Schwarz S, et al. Head-to-head comparisons of metabolic side effects of second generation antipsychotics in the treatment of schizophrenia: a systematic review and meta-analysis. Schizophr Res. 2010;123(2-3):225-233.

9. Rummel-Kluge C, Komossa K, Schwarz S, et al. Second-generation antipsychotic drugs and extrapyramidal side effects: a systematic review and meta-analysis of head-to-head comparisons. Schizophr Bull. 2012;38(1):167-177.

10. National Institute for Health and Care Excellence. Psychosis and Schizophrenia in Adults: Treatment and Management. London: NICE; 2014

11. Topamax (topiramate) [package insert]. Beerse, Belgium: Janssen Pharmaceutica; 2005.

12. Hahn MK, Cohn T, Teo C, Remington G. Topiramate in schizophrenia: a review of effects on psychopathology and metabolic parameters. Clin Schizophr Relat Psychoses. 2013;6(4):186-196.

13. Wilkes JJ, Nelson E, Osborne M, Demarest KT, Olefsky JM. Topiramate is an insulin-sensitizing compound in vivo with direct effects on adipocytes in female ZDF rats. Am J Physiol Endocrinol Metab. 2005;288(3):E617-E624.

14. Berelowitz M, Matthews J, Pimstone BL, Kronheim S, Sacks H. Immunoreactive somatostatin in rat cerebral cortical and hypothalamic synaptosomes. Metabolism. 1978;27(9 Suppl 1):1171-1173.

15. Afshar H, Roohafza H, Mousavi G, et al. Topiramate add-on treatment in schizophrenia: a randomised, double-blind, placebo-controlled clinical trial. J Psychopharmacol. 2009;23(2):157-162.

16. Behdani F, Hebrani P, Ardani AR, Rafee E. Effect of topiramate augmentation in chronic schizophrenia: a placebo-controlled trial. Arch Iran Med. 2011;14(4):270-275.

17. Chengappa KN, Kupfer DJ, Parepally H, et al. A placebo-controlled, random-assignment, parallel-group pilot study of adjunctive topiramate for patients with schizoaffective disorder, bipolar type. Bipolar Disord 2007;9(6):609-617.

18. Kim JH, Yim SJ, Nam JH. A 12-week, randomized, open-label, parallelgroup trial of topiramate in limiting weight gain during olanzapine treatment in patients with schizophrenia. Schizophr Res. 2006;82(1): 115-117.

19. Ko YH, Joe SH, Jung IK, Kim SH. Topiramate as an adjuvant treatment with atypical antipsychotics in schizophrenic patients experiencing weight gain. Clin Neuropharmacol. 2005;28(4):169-175.

20. Muscatello MR, Bruno A, Pandolfo G, et al. Topiramate augmentation of clozapine in schizophrenia: a double-blind, placebo-controlled study. J Psychopharmacol. 2011;25(5):667-674.

21. Narula PK, Rehan HS, Unni KE, Gupta N. Topiramate for prevention of olanzapine associated weight gain and metabolic dysfunction in schizophrenia: a double-blind, placebo-controlled trial. Schizophr Res. 2010;118(1-3):218-223.

22. Nickel MK, Nickel C, Muehlbacher M, et al. Influence of topiramate on olanzapine-related adiposity in women: a random, double-blind, placebocontrolled study. J Clin Psychopharmacol. 2005;25(3):211-217.

23. Tiihonen $\mathrm{J}$, Halonen $\mathrm{P}$, Wahlbeck $\mathrm{K}$, et al. Topiramate add-on in treatment-resistant schizophrenia: a randomized, double-blind, placebocontrolled, crossover trial. J Clin Psychiatry. 2005;66(8):1012-1015.

24. Chen ZX, Xia ML, Xia WJ, Huang HG, Jin L. [Topiramatefor prevention of olanzapine associated weight gain]. Modern Pract Med. 2012; 24:979-980. Chinese.
25. Liu JJ. [The efficacy of topiramate in the treatment of antipsychoticinduced weight gain and lipid disorders]. Modern Pract Med. 2011; 23:665-667. Chinese.

26. Yang Y. The studying about the effects of topiramate in preventing olanzapine-induced weight gain and glucose and lipid metabolic dysfunction [dissertation]. Chongqing: Chongqing Medical University; 2008. Available at: http://cdmd.cnki.com.cn/Article/CDMD-106312008176780.htm.dissertation.

27. Sommer IE, Begemann MJ, Temmerman A, Leucht S. Pharmacological augmentation strategies for schizophrenia patients with insufficient response to clozapine: a quantitative literature review. Schizophr Bull. 2012;38(5):1003-1011.

28. Mizuno Y, Suzuki T, Nakagawa A, et al. Pharmacological strategies to counteract antipsychotic-induced weight gain and metabolic adverse effects in schizophrenia: a systematic review and meta-analysis. Schizophr Bull. 2014;40(6):1385-1403.

29. Moher D, Liberati A, Tetzlaff J, Altman DG. Preferred reporting items for systematic reviews and meta-analyses: the PRISMA statement. BMJ. 2009;339:b2535

30. Kay SR, Fiszbein A, Opler LA. The positive and negative syndrome scale (PANSS) for schizophrenia. Schizophr Bull. 1987;13(2):261-276.

31. Guy W, Bonato RR. Manual for the ECDEU Assessment Battery. 2nd ed. Chevy Chase (MD): National Institute of Mental Health; 1970.

32. Overall J, Gorham D. The brief psychiatric rating scale. Psychol Rep. 1962;10:799-812.

33. Andreasen N. Scale for the Assessment of Positive Symptoms (SAPS). Iowa City: University of Iowa; 1984.

34. Andreasen N. Scale for the Assessment of Negative Symptoms (SANS). Iowa City: University of Iowa; 1984.

35. Addington D, Addington J, Schissel B. A depression rating scale for schizophrenics. Schizophr Res. 1990;3(4):247-251.

36. Montgomery SA, Asberg M. A new depression scale designed to be sensitive to change. Br J Psychiatry. 1979;134:382-389.

37. DerSimonian R, Laird N. Meta-analysis in clinical trials. Control Clin Trials. 1986;7(3):177-188.

38. Higgins JP, Thompson SG, Deeks JJ, Altman DG. Measuring inconsistency in meta-analyses. BMJ. 2003;327(7414):557-560.

39. Liang H, Li H, Hu Y, Li S, Lu L, Song X. [Effects of topiramate for atypical antipsychotic-induced body weight gain and metabolic adversities: a systematic review and meta-analysis]. Zhonghua Yi Xue Za Zhi. 2016;96(3):216-223. Chinese.

40. De Hert MA, van Winkel R, Van Eyck D, et al. Prevalence of the metabolic syndrome in patients with schizophrenia treated with antipsychotic medication. Schizophr Res. 2006;83(1):87-93.

41. Kishi T, Mukai T, Matsuda Y, Moriwaki M, Iwata N. Efficacy and safety of noradrenalin reuptake inhibitor augmentation therapy for schizophrenia: a meta-analysis of double-blind randomized placebocontrolled trials. J Psychiatr Res. 2013;47(11):1557-1563.

42. Kishi T, Meltzer HY, Iwata N. Augmentation of antipsychotic drug action by azapirone 5-HT1A receptor partial agonists: a meta-analysis. Int J Neuropsychopharmacol. 2013;16(6):1259-1266.

43. Brain C, Sameby B, Allerby K, et al. Twelve months of electronic monitoring (MEMS) in the Swedish COAST-study: a comparison of methods for the measurement of adherence in schizophrenia. Eur Neuropsychopharmacol. 2014;24(2):215-222.

44. Moritz S, Hünsche A, Lincoln TM. Nonadherence to antipsychotics: the role of positive attitudes towards positive symptoms. Eur Neuropsychopharmacol. 2014;24(11):1745-1752.

45. Leucht S, Corves C, Arbter D, Engel RR, Li C, Davis JM. Secondgeneration versus first-generation antipsychotic drugs for schizophrenia: a meta-analysis. Lancet. 2009;373(9657):31-41.

46. Woodhead M. $80 \%$ of China's clinical trial data are fraudulent, investigation finds. BMJ. 2016;355:i5396.

47. Correll CU, Maayan L, Kane J, Hert MD, Cohen D. Efficacy for psychopathology and body weight and safety of topiramate-antipsychotic cotreatment in patients with schizophrenia spectrum disorders: results from a meta-analysis of randomized controlled trials. J Clin Psychiatry. 2016;77:e746-e756. 


\section{Supplementary materials}

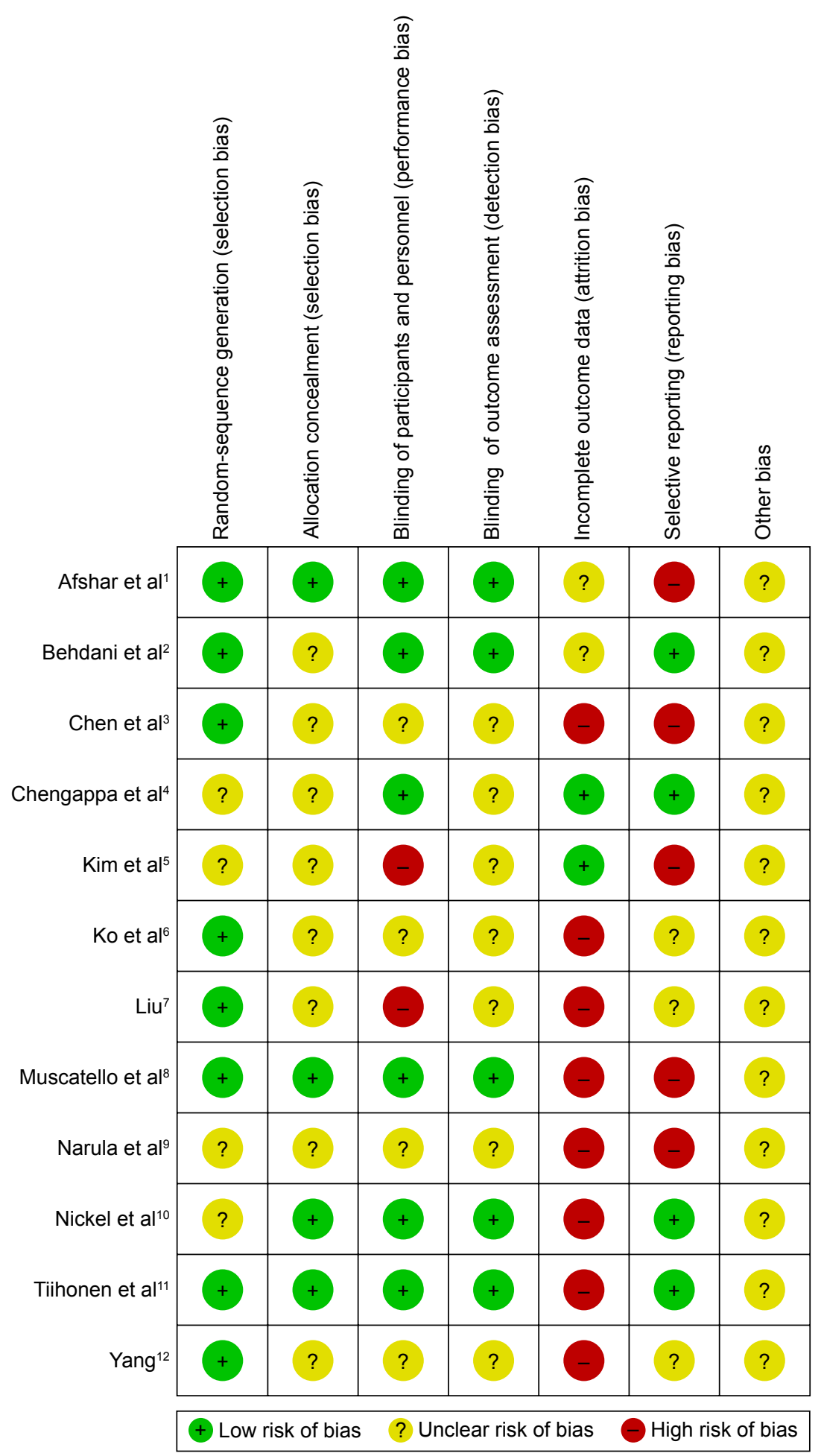

Figure SI Risk-of-bias summary. 
Random-sequence generation (selection bias)

Allocation concealment (selection bias)

Blinding of participants and personnel (performance bias)

Blinding of outcome assessment (detection bias)

Incomplete outcome data (attrition bias)

Selective reporting (reporting bias)

Other bias

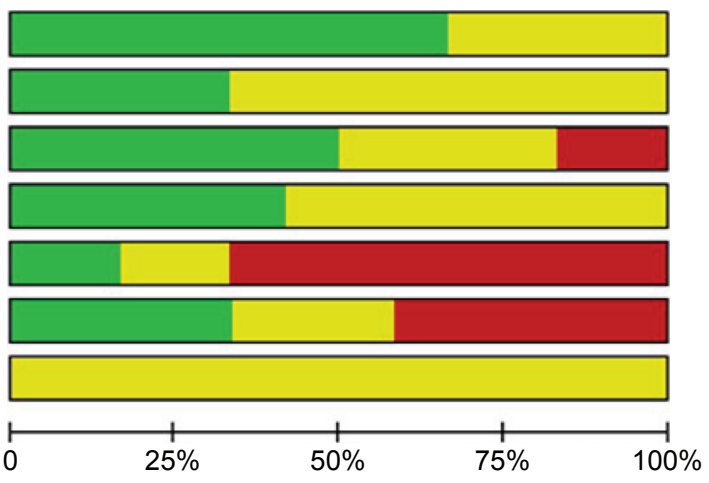

Low risk of bias $\quad \square$ Unclear risk of bias $\quad \square$ High risk of bias

Figure S2 Risk-of-bias graph.

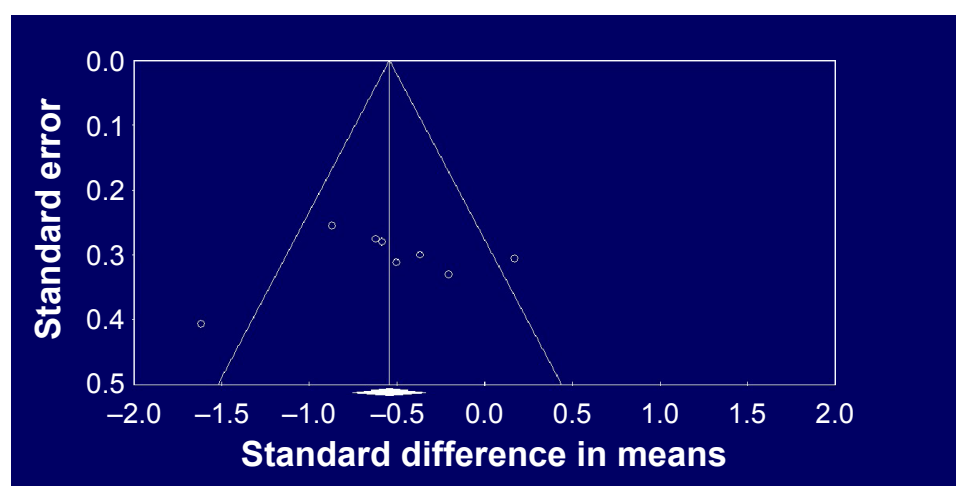

Figure S3 Funnel plot of standard error by standard difference in means.

\section{References}

1. Afshar H, Roohafza H, Mousavi G, et al. Topiramate add-on treatment in schizophrenia: a randomised, double-blind, placebo-controlled clinical trial. J Psychopharmacol. 2009;23(2):157-162.

2. Behdani F, Hebrani P, Ardani AR, Rafee E. Effect of topiramate augmentation in chronic schizophrenia: a placebo-controlled trial. Arch Iran Med. 2011;14(4):270-275.

3. Chen ZX, Xia ML, Xia WJ, Huang HG, Jin L. [Topiramatefor prevention of olanzapine associated weight gain]. Modern Pract Med. 2012; 24:979-980. Chinese.

4. Chengappa KN, Kupfer DJ, Parepally H, et al. A placebo-controlled, random-assignment, parallel-group pilot study of adjunctive topiramate for patients with schizoaffective disorder, bipolar type. Bipolar Disord. 2007;9(6):609-617.

5. Kim JH, Yim SJ, Nam JH. A 12-week, randomized, open-label, parallelgroup trial of topiramate in limiting weight gain during olanzapine treatment in patients with schizophrenia. Schizophr Res. 2006;82(1):115-117.

6. Ko YH, Joe SH, Jung IK, Kim SH. Topiramate as an adjuvant treatment with atypical antipsychotics in schizophrenic patients experiencing weight gain. Clin Neuropharmacol. 2005;28(4):169-175.

7. Liu JJ. [The efficacy of topiramate in the treatment of antipsychoticinduced weight gain and lipid disorders]. Modern Pract Med. 2011;23: 665-667. Chinese.
8. Muscatello MR, Bruno A, Pandolfo G, et al. Topiramate augmentation of clozapine in schizophrenia: a double-blind, placebo-controlled study. J Psychopharmacol. 2011;25(5):667-674.

9. Narula PK, Rehan HS, Unni KE, Gupta N. Topiramate for prevention of olanzapine associated weight gain and metabolic dysfunction in schizophrenia: a double-blind, placebo-controlled trial. Schizophr Res. 2010;118(1-3):218-223.

10. Nickel MK, Nickel C, Muehlbacher M, et al. Influence of topiramate on olanzapine-related adiposity in women: a random, double-blind, placebocontrolled study. J Clin Psychopharmacol. 2005;25(3):211-217.

11. Tiihonen J, Halonen P, Wahlbeck K, et al. Topiramate add-on in treatment-resistant schizophrenia: a randomized, double-blind, placebo-controlled, crossover trial. J Clin Psychiatry. 2005;66(8): 1012-1015.

12. Yang Y. The studying about the effects of topiramate in preventing olanzapine-induced weight gain and glucose and lipid metabolic dysfunction [dissertation]. Chongqing: Chongqing Medical University; 2008. Available at: http://cdmd.cnki.com.cn/Article/CDMD-106312008176780.htm.dissertation. 


\section{Publish your work in this journal}

Neuropsychiatric Disease and Treatment is an international, peerreviewed journal of clinical therapeutics and pharmacology focusing on concise rapid reporting of clinical or pre-clinical studies on a range of neuropsychiatric and neurological disorders. This journal is indexed on PubMed Central, the 'PsycINFO' database and CAS,

and is the official journal of The International Neuropsychiatric Association (INA). The manuscript management system is completely online and includes a very quick and fair peer-review system, which is all easy to use. Visit http://www.dovepress.com/testimonials.php to read real quotes from published authors.

Submit your manuscript here: http://www.dovepress.com/neuropsychiatric-disease-and-treatment-journal 